\title{
BMJ Open Outdoor green space exposure and brain health measures related to Alzheimer's disease: a rapid review
}

\author{
Lilah Besser
}

To cite: Besser L. Outdoor green space exposure and brain health measures related to Alzheimer's disease: a rapid review. BMJ Open 2021;11:e043456. doi:10.1136/ bmjopen-2020-043456

- Prepublication history and supplemental material for this paper is available online. To view these files, please visit the journal online (http://dx.doi org/10.1136/bmjopen-2020043456).

Received 04 August 2020 Revised 17 March 2021 Accepted 09 April 2021

Check for updates

(C) Author(s) (or their employer(s)) 2021. Re-use permitted under CC BY-NC. No commercial re-use. See rights and permissions. Published by BMJ.

Institute for Human Health and Disease Intervention, Florida Atlantic University, Boca Raton, Florida, USA

Correspondence to

Dr Lilah Besser;

Ibesser@fau.edu

\section{ABSTRACT}

Objectives Summarise studies of outdoor green space exposure and brain health measures related to Alzheimer's disease and related disorders (ADRD), and determine scientific gaps for future research.

Design Rapid review of primary research studies. Methods and outcomes PubMed, Embase and Web of Science Core Collection were searched for articles meeting the criteria published on/before 13 February 2020. The review excluded papers not in English, focused on transient states (eg, mental fatigue) or not using individual-level measures of brain health (eg, average school test scores). Brain health measures of interest included cognitive function, clinical diagnosis of cognitive impairment/dementia/ADRD and brain biomarkers such as those from MRI, measures typically associated with ADRD risk and disease progression.

Results Twenty-two papers were published from 2012 to $2020,36 \%$ on $<18$ years old, $32 \%$ on $18-64$ years old and $59 \%$ on $\geq 65$ years old. Sixty-four per cent defined green space based on the Normalised Difference Vegetation Index ('greenness'/healthy vegetation) and $68 \%$ focused on cognitive measures of brain health (eg, memory). Seventeen studies (77\%) found green space-brain health associations (14 positive, 4 inverse). Greater greenness/ green space was positively associated various cognitive domains in 10 studies and with MRI outcomes (regional brain volumes, cortical thickness, amygdala integrity) in three studies. Greater neighbourhood greenness was associated with lower odds/risk of cognitive impairment/ ADRD in some studies but increased odds/risk in others ( $n=4$ studies).

Conclusions Published studies suggest positive green space-brain health associations across the life course, but the methods and cohorts were limited and heterogeneous. Future research using racially/ethnically and geographically diverse cohorts, life course methods and more specific green space and brain health measures (eg, time spent in green spaces, ADRD biomarkers) will strengthen evidence for causal associations.

\section{INTRODUCTION}

Nature contact involves time spent in green spaces (eg, gardens, parks, forests) and blue spaces (eg, lakes, rivers) where people live, work and play. Preliminary studies suggest associations between nature contact and health including reductions in depression,

\section{Strengths and limitations of this study}

Three major databases covering biomedical, psychological, environmental and social science topics and a range of keywords were searched to find pertinent studies regarding associations between green space exposure and Alzheimer's disease and related disorders brain health measures.

- Published literature reviews on green space and health and reference lists from the final sample of papers were reviewed to help ensure pertinent papers were included.

- This study was limited to a single reviewer and thus, the methods used to search, screen, select and chart the final sample of papers could not be duplicated/adjudicated by additional reviewers.

- As a rapid review, this study was not aimed at providing a quantitative evaluation of the evidence or risk of bias, and may have missed papers that would have been ascertained if additional reviewers were available.

anxiety and cardiovascular risk factors; improved attention and mood; and increased physical activity. ${ }^{1}$ Studies also suggest associations with brain health across the life course. $^{2-8}$ For instance, greater neighbourhood greenness (ie, healthy vegetation) has been associated with lower odds of Alzheimer's disease (AD) in older adults. ${ }^{9}$

$\mathrm{AD}$ and related disorders (ADRD) affect approximately 50 million people worldwide, and $15 \%$ of older adults have mild cognitive impairment, a frequent antecedent to dementia. ${ }^{10} 11$ Older age, lower educational attainment and genetics (eg, apolipoprotein $\mathrm{E}$ (APOE) $\varepsilon 4$ allele carriers) are some of the strongest predictors of $\mathrm{AD}$ risk and late-life cognitive decline. ${ }^{12}$ Clinicians diagnose $\mathrm{AD}$ using biomarkers and/or cognitive assessments. Diagnostic biomarkers include cerebrospinal fluid (CSF) or positron emission (PET) scan biomarkers measuring brain amyloid beta and phosphorylated tau, the proteins responsible for AD neuropathology (ie, plaques and tangles). ${ }^{13} 14$ Cognitive tests 
for $\mathrm{AD}$ typically evaluate memory of personal events (ie, episodic memory), the hallmark cognitive domain affected early in the disease course. ${ }^{15}$ Episodic memory problems are correlated with atrophy of the hippocampus, and thus, MRI brain biomarkers such as hippocampal atrophy help support $\mathrm{AD}$ diagnosis and predict $\mathrm{AD}$ incidence and disease progression. ${ }^{16}$ Other dementia disorders typically affect different cognitive domains/ brain regions in the early stages of disease, and later stages of ADRD can affect additional cognitive domains and brain regions. ${ }^{15}$

The psychological and financial burden of ADRD on patients and families is substantial. ${ }^{1718}$ Healthcare systems are ill prepared to deal with the increase in ADRD prevalence accompanying the rapidly rising population of older adults, ${ }^{19}$ and no effective treatments are currently available. Therefore, an accumulating body of research has focused on individual-level and community-level interventions that may be help prevent or delay ADRD. Provided there is supporting evidence, neighbourhood green space is one such community-level feature that may be promoted to improve lifelong brain health. Healthy brain development during childhood and maintenance of brain health throughout adulthood, assisted by living near health-enhancing green spaces, may help reduce ADRD risk.

Green space exposure may benefit brain health through a number of pathways. ${ }^{120}$ They provide enriching, physical activity promoting and stress reducing environments that consequently may be associated with better brain health by affecting cerebral blood flow, angiogenesis, vascular integrity, cell proliferation/survival, vascular dysregulation and/or inflammation. ${ }^{21-25}$ Green space exposure may reduce stress and mental fatigue and improve attention, consistent with the stress recovery theory and attention restoration theory. ${ }^{26-28}$ Studies are available to support both theories. For instance, living within one mile of green spaces and visiting green spaces have been associated with experiencing less stress, ${ }^{29}$ and gardening has been found to reduce levels of salivary cortisol, a stress hormone. ${ }^{30}$ In adults, mood, restoration and sustained attention were improved after participating in a nature walk intervention in urban and rural locales. ${ }^{28}$ These psychological benefits over the long term may additionally benefit mental health (eg, anxiety, depression), factors associated with brain health including ADRD risk. ${ }^{31}$ Microbial and antigenic exposures from nature contact, ${ }^{32}$ especially during childhood, may affect lifelong immune function and contribute to healthy microbiomes, which have been associated with mental health and $\mathrm{AD} .{ }^{33-35}$ Green spaces provide areas for recreational exercise. Exposure and access to natural places have been associated with greater physical activity in children through older adults, ${ }^{3637}$ and obtaining greater physical activity has been associated with reduced brain atrophy, cognitive decline and ADRD risk. ${ }^{38}{ }^{39}$ Natural areas provide spaces for social gathering and engagement. ${ }^{40}$ Higher levels of social engagement have been associated with better cognitive function and reduced $\mathrm{AD}$ risk. ${ }^{41}{ }^{42}$ Lastly, natural areas and parks have been associated with lower levels of harmful air pollutants, including particular matter $\leq 10 \mu \mathrm{m}$ in size $\left(\mathrm{PM}_{10}\right)$ and nitrogen dioxide $\left(\mathrm{NO}_{2}\right)^{43} 44$ that have been associated with worse cognition and greater ADRD risk. ${ }^{45}$ The mechanisms by which air pollution affects the brain have been hypothesised to be direct and/or indirect (eg, systemic inflammation, adsorbed compounds). ${ }^{46}$

The budding and cross-disciplinary field of research on green spaces and ADRD/brain health will benefit from a review of pertinent studies spanning multiple disciplines. Literature used to inform primary research tends to be siloed to a researcher's area of expertise or based on limited or discipline-specific search terms. Given the nascent state of green space and ADRD-related brain health research and the lack of published literature reviews focused on the topic, this rapid review employed scoping aims. Rapid reviews are increasingly used in research to address the need for more readily available summaries of available evidence that cannot be achieved through the lengthy and resource-intensive process of systematic reviews. ${ }^{47}$ Scoping reviews are useful in summarising new topics of research, findings for a broader set of health outcomes, or topics that may not have enough evidence amassed to assess the weight of evidence or risk of bias. ${ }^{47-49}$

The number of studies on green space and health has risen dramatically in the last decade, ${ }^{50}$ but it remains unclear how many studied brain health outcomes. Therefore, consistent with the major goals of a scoping review, 495152 this rapid review aimed: (1) to summarise the extant literature on green space-brain health associations across the life course, potentially providing impetus for future systematic reviews and (2) to identify knowledge gaps to inform future research. The primary intent was to identify and describe current evidence for benefits to cognition and brain structure/function due to green space exposure. These benefits may develop and persist in early- and mid-life to reduce ADRD risk in late life.

\section{METHODS}

\section{Patient and public involvement}

Patients and the public were not involved as this study focuses on a review of published papers with no analysis of participant data.

\section{Identification and study selection}

A single reviewer was available for this study. On 13 February 2020, PubMed, Web of Science Core Collection and Embase were queried for the following keywords: 'greenspace or green space or greenness or parks or park or park space or parkspace' and 'cognition or cognitive or memory or brain ageing or Alzheimer or Alzheimer's or dementia or cognitive impairment'. To help ensure the 13 February review did not miss pertinent papers, a second search of the three databases was performed on 18 July 2020, for the following keywords: 'neighbourhood environment or wilderness or greenery or natural space 
or natural environment or public garden or recreational resource or Normalised Difference Vegetation Index (NDVI) or built environment or open space or woodland' and 'brain volume or brain atrophy or neurodegenerative disease or Alzheimer biomarker or cognition or cognitive or memory or brain ageing or Alzheimer or Alzheimer's or dementia or cognitive impairment'. The keywords searched reflected the brain health measures of interest that are typically associated with ADRD risk/ disease progression, including cognitive function, clinical diagnosis of cognitive impairment/dementia/ADRD and biomarkers such as those from brain imaging (eg, MRI).

The 18 July 2020 search was restricted to papers published on or before 13 February, 2020, to be consistent with the original search. A limitation of the 18 July 2020 search was the restriction to a search of titles in Web of Science. A full-text search led to 8574 papers that could not be feasibly reviewed based on available time and resources (ie, this is a rapid review). Of note, the final list of included papers from the February 13 search was ascertained either from the search of PubMed and Embase or the review of resulting titles from the search of full texts in Web of Science (ie, not from a full text review of papers in Web of Science). This suggests that the July search of titles in Web of Science was unlikely to have missed pertinent papers, but the possibility remains. A detailed description of the search strategy is provided in online supplemental figure 1 .

Titles were screened for topics definitely or possibly related to green space and ADRD-related brain health. Titles potentially related were included in the abstract review (eg, green space and child development, neighbourhood environment and $\mathrm{AD}$, built environments and ageing, outdoors and mental health). After review, abstracts that moved on to full-text review had exposures/outcomes directly pertinent to this study, focused on associations between green space and other measures but mentioned brain health measures as covariates, or seemed possibly relevant by including closely related exposures or outcomes (eg, mental health, frailty, built environment, nature contact). Full texts included in the final sample reported associations between green space exposure and brain health outcomes in the main text or online supplemental file.

Articles were excluded if they: (1) were not in English; (2) were not primary research studies; (3) were focused on indoor green space/views; (4) used virtual reality to simulate green spaces; (5) were ecological studies (eg, average school test scores); (6) were focused on attention restoration or mental fatigue (transient states) or (7) centred on green space activities such as gardening without an adequate control/comparison group to sufficiently capture green space as the main exposure. Reference lists from the final sample and published green space-health reviews were reviewed to identify other studies meeting the eligibility criteria. ${ }^{1-8}$



Figure 1 Sample size flow diagram (see online supplemental figure 1 for full details).

\section{Charting and summarising the data}

Papers were described by study design, location, age groups, green space and brain health measures and definitions, statistical methods and main findings (these data were charted into online supplemental tables 1-4). Key study elements were tabulated separately for three major age groups: children (0-17 years), adults (18-64 years) and older adults ( $\geq 65$ years). Findings were stratified by age because while studies of children focus on the critical period of childhood development, studies of 18-64 years old focus on working adults and studies of $\geq 65$ years old focus on retirement-age individuals. Green space exposures and brain health can differ substantially during these life stages. Results (positive, inverse, null associations) were summarised according to age groups, green space measures, brain health measures and examined green space-brain health associations to characterise the scope of the evidence to date.

\section{RESULTS}

\section{Overall study characteristics}

The final sample included 22 papers (figure 1). ${ }^{9}$ 53-73 Post hoc additions to the final sample, published on or before 13 February 2020, included one paper previously known by the author ${ }^{53}$ and one paper identified from the final sample reference lists. ${ }^{73}$ Tables $1-4$ and online supplemental tables 1-4 summarise study characteristics and findings. Eight-two per cent $(\mathrm{n}=18)^{954-5860-6466-6971-73}$ of studies were published on/after 2017 (range: 2012$2020)$. Seven studies $(32 \%)$ were in the UK, four $(18 \%)$ in China, three in Spain (14\%), two each (9\%) in the USA and Canada and one each (4\%) in Bulgaria, Germany, New Zealand and multiple regions (Spain, UK, the Netherlands) (figure 2). Eight studies $(36 \%)$ focused on $<18$ 
Table 1 Summary of green space-brain health associations by age group

\begin{tabular}{|c|c|c|c|c|c|c|}
\hline Citation* & Sample size & $\begin{array}{l}\text { Population based/ } \\
\text { random sample }\end{array}$ & Location & $\begin{array}{l}\text { Children } \\
\text { (<18 years) }\end{array}$ & $\begin{array}{l}\text { Adults } \\
\text { (18-64 years) }\end{array}$ & $\begin{array}{l}\text { Older adults } \\
\text { ( } \geq 65 \text { years) }\end{array}$ \\
\hline Brown ${ }^{9}$ & 249405 & Yes & USA & & & + \\
\hline Cherrie $^{63}$ & 281 & Yes & UK & $\mathrm{N}$ & $\mathrm{N}$ & + \\
\hline Cherrie $^{64}$ & 281 & Yes & UK & & & $+\mathrm{N}$ \\
\hline Clarke $^{53}$ & 949 & Yes & USA & & $\mathrm{N}$ & $\mathrm{N}$ \\
\hline Dadvand $^{65}$ & 2593 & No & Spain & $+\mathrm{N}$ & & \\
\hline Dadvand ${ }^{54}$ & 987 & Yes & Spain & $+\mathrm{N}$ & & \\
\hline Dadvand ${ }^{66}$ & 253 & No & Spain & $+\mathrm{N}$ & & \\
\hline Dzhambov $^{72}$ & 112 & No & Bulgaria & & $+\mathrm{N}$ & \\
\hline de Keijzer ${ }^{55}$ & 6506 & Yes & UK & & $+\mathrm{N}$ & $+\mathrm{N}$ \\
\hline Flouri ${ }^{56}$ & 4758 & Yes & UK & + & & \\
\hline Hystad $^{57}$ & 6658 & Yes & Canada & & $-N$ & \\
\hline Kuhn $^{73}$ & 341 & No & Germany & & & $+\mathrm{N}$ \\
\hline $\operatorname{Liao}^{67}$ & 1312 & No & China & + & & \\
\hline Reuben $^{68}$ & 1658 & Yes & UK & $\mathrm{N}$ & & \\
\hline Wang $^{69}$ & 3544 & No & China & & & $\mathrm{N}$ \\
\hline Ward $^{70}$ & 72 & No & New Zealand & $\mathrm{N}$ & & \\
\hline$W u^{59}$ & 2424 & Yes & UK & & & - \\
\hline$W^{58}$ & 7505 & Yes & UK & & & $-N$ \\
\hline $\mathrm{Yu}^{71}$ & 3240 & No & China & & & $\mathrm{N}$ \\
\hline Yuchi $^{60}$ & 678000 & Yes & Canada & & +- & +- \\
\hline Zhu $^{61}$ & 6994 & Yes & China & & & + \\
\hline Zijlema ${ }^{62}$ & 1628 & Yes & $\begin{array}{l}\text { Spain, UK, } \\
\text { Netherlands }\end{array}$ & & $+\mathrm{N}$ & $+\mathrm{N}$ \\
\hline \multicolumn{3}{|c|}{ Studies with positive associations } & & 5 & 4 & 8 \\
\hline \multicolumn{3}{|c|}{ Studies with inverse associations } & & 0 & 2 & 3 \\
\hline \multicolumn{3}{|c|}{ Studies with null associations } & & 6 & 6 & 8 \\
\hline \multicolumn{3}{|l|}{ Total studies } & & 8 & 7 & 13 \\
\hline
\end{tabular}

${ }^{*}$ Full list of papers found in online supplemental text 1.

+, positive association; -, inverse association; N, null association.

years old (childhood) ${ }^{54566365-6870}$ seven $(32 \%)$ focused on 18-64 years old (adulthood), 53555760626372 and $13(59 \%)$ focused on $\geq 65$ years old (older adulthood) ${ }^{9535558-64697173}$ (figure 3). Fourteen studies $(64 \%)^{953-6468}$ were based on population-based cohorts or random sampling strategies. Two studies (9\%) examined life course associations, both investigating childhood and mid-life park space exposures and cognitive change in late life..$^{634}$

Seventeen studies $(77 \%)$ found associations (14 positive, $^{9}{ }^{54-56} 60-6772734$ inverse $^{57-60}$ ) and 5 (23\%) found no associations ${ }^{53}{ }^{68-71}$ between greenness/green space and brain health (tables 1-4, figure 4). Twelve studies $(55 \%)$ reported a combination of positive, inverse and/ or null associations. ${ }^{5455} 57586062-667273$ All but one study ${ }^{69}$ employed multivariable linear or logistic regression accounting for key confounders (ie, age, sex, socioeconomic status (SES)) and $12(55 \%)^{9} 53-5658596162646570$ used regression methods accounting for data clustering/ multilevel data.

\section{Findings by age group}

Children: Five ${ }^{54} 5665-67$ of the eight studies ${ }^{54} 566365-6870$ found green space-brain health associations in children (five positive, zero inverse) (table 1). Greater neighbourhood greenness/green space was associated with working memory, ${ }^{546}$ attention ${ }^{5465}$ and intellectual development ${ }^{67}$ and with specific brain regions. ${ }^{66}$ Null associations were found between greater greenness/green space and intelligence, ${ }^{63}$ alerting, ${ }^{65}$ orienting, ${ }^{65}$ executive processing/ function, ${ }^{65} 68$ fluid ability, ${ }^{68}$ crystallised ability, ${ }^{68}$ working memory ${ }^{68}$ and attention. ${ }^{56568}$ Time spent in green space measured via global positioning system (GPS) tracking was not associated with multiple cognitive domains (eg, visual and verbal memory, processing speed).$^{70}$ 
Table 2 Summary of green space-brain health associations by green space measure

\begin{tabular}{|c|c|c|c|c|c|c|c|c|c|c|}
\hline Citation* & $\begin{array}{l}\text { Sample } \\
\text { size }\end{array}$ & $\begin{array}{l}\text { Population } \\
\text { based/ } \\
\text { random } \\
\text { sample }\end{array}$ & Location & $\begin{array}{l}\text { Longitudinal } \\
\text { green space }\end{array}$ & $\begin{array}{l}\text { Greenness } \\
\text { (NDVI, EVI) }\end{array}$ & $\begin{array}{l}\text { Percent/ } \\
\text { area } \\
\text { park } \\
\text { space }\end{array}$ & $\begin{array}{l}\text { Percent } \\
\text { green } \\
\text { space }\end{array}$ & $\begin{array}{l}\text { Time } \\
\text { spent } \\
\text { in } \\
\text { green } \\
\text { space }\end{array}$ & $\begin{array}{l}\text { Distance } \\
\text { to natural } \\
\text { outdoor } \\
\text { environment }\end{array}$ & $\begin{array}{l}\text { Other } \\
\text { green } \\
\text { space }\end{array}$ \\
\hline Brown ${ }^{9}$ & 249405 & Yes & USA & No & + & & & & & \\
\hline Cherrie $^{63}$ & 281 & Yes & UK & Yes & & $+\mathrm{N}$ & & & & \\
\hline Dadvand $^{65}$ & 2593 & No & Spain & No & $+\mathrm{N}$ & & & & & \\
\hline Dadvand $^{54}$ & 987 & Yes & Spain & Yes & $+\mathrm{N}$ & & & & & \\
\hline Dadvand $^{66}$ & 253 & No & Spain & Yes & $+\mathrm{N}$ & & & & & \\
\hline Dzhambov $^{72}$ & 112 & No & Bulgaria & No & $+\mathrm{N}$ & & & & & \\
\hline Kuhn $^{73}$ & 341 & No & Germany & No & & & $+\mathrm{N}$ & & & \\
\hline $\operatorname{Liao}^{67}$ & 1312 & No & China & No & + & & & & & \\
\hline Reuben ${ }^{68}$ & 1658 & Yes & UK & Yes & $\mathrm{N}$ & & & & & \\
\hline Wang ${ }^{69}$ & 3544 & No & China & No & $\mathrm{N}$ & & & & & \\
\hline Ward $^{70}$ & 72 & No & New Zealand & No & & & & $\mathrm{N}$ & & \\
\hline $\mathrm{Wu}^{59}$ & 2424 & Yes & UK & No & & & - & & & \\
\hline$W u^{58}$ & 7505 & Yes & UK & No & & & $-N$ & & & \\
\hline $\mathrm{Yu}^{71}$ & 3240 & No & China & No & $\mathrm{N}$ & & & & & \\
\hline Yuchi $^{60}$ & 678000 & Yes & Canada & Yes & +- & & & & & \\
\hline \multicolumn{5}{|l|}{ Total studies } & 14 & 3 & 4 & 2 & 1 & 1 \\
\hline
\end{tabular}

${ }^{*}$ Full list of papers found in online supplemental text 1.

-, inverse association; +, positive association; EVI, enhanced vegetation index; N, null association; NDVI, Normalised Difference Vegetation Index.

Adults (18-64 years): Five of the seven studies 535557606372 found green space-brain health associations in adults (four positive, ${ }^{55606272}$ two inverse ${ }^{5760}$ (table 1). Increased residential distance to natural outdoor environments was associated with longer cognitive test completion times, ${ }^{62}$ and greater neighbourhood greenness was positively and inversely associated with dementia diagnoses (detailed in 'older adults' section below) ${ }^{60}$ Greater neighbourhood greenness was cross-sectionally associated with better global cognition ${ }^{72}$ and was associated with slower longitudinal decline on global cognition, reasoning and verbal fluency. ${ }^{55}$ Additionally, greater neighbourhood green space was associated with greater cortical thickness in the prefrontal cortex, bilateral fusiform gyrus, left precuneus and insula, and right cuneus as measured via MRI. ${ }^{72}$ Null associations were found between greater neighbourhood greenness/green space or 5-year change in greenness and measures of global cognition, ${ }^{53} 72$ intelligence, ${ }^{63}$ reaction time,${ }^{57}$ reasoning, ${ }^{57}$ memory, ${ }^{55} 5772$ naming $^{72}$ and visual attention/executive processing. ${ }^{62}$ No associations were found between self-reported visits or time spent in natural environments and visual attention/ executive processing, ${ }^{62}$ and no associations were observed between greater greenness and cortical thickness of other brain MRI regions (eg, right cuneus and insula). ${ }^{72}$ Lastly, inverse associations were found between 5-year change in neighbourhood greenness and reasoning. ${ }^{57}$

Older adults ( $\geq 65$ years). Ten of 13 studies ${ }^{9535558-646971} 73$ found green space-brain health associations in older adults (eight positive, ${ }^{955} 60-6473$ three inverse ${ }^{58-60}$ (table 1). 
Table 3 Summary of green space-brain health associations by brain health measure

\begin{tabular}{|c|c|c|c|c|c|c|c|}
\hline Citation* & $\begin{array}{l}\text { Sample } \\
\text { size }\end{array}$ & $\begin{array}{l}\text { Population } \\
\text { based / } \\
\text { random } \\
\text { sample }\end{array}$ & Location & $\begin{array}{l}\text { Longitudinal } \\
\text { brain health } \\
\text { measure }\end{array}$ & Cognition & $\begin{array}{l}\text { MRI brain } \\
\text { regions }\end{array}$ & $\begin{array}{l}\text { Diagnosis } \\
\text { of cognitive } \\
\text { impairment/ } \\
\text { dementia }\end{array}$ \\
\hline Brown ${ }^{9}$ & 249405 & Yes & USA & No & & & + \\
\hline Cherrie $^{63}$ & 281 & Yes & UK & Yes & $+\mathrm{N}$ & & \\
\hline Cherrie $^{64}$ & 281 & Yes & UK & Yes & $+\mathrm{N}$ & & \\
\hline Clarke ${ }^{53}$ & 949 & Yes & USA & No & $\mathrm{N}$ & & \\
\hline Dadvand $^{65}$ & 2593 & No & Spain & Yes & $+\mathrm{N}$ & & \\
\hline Dadvand $^{54}$ & 987 & Yes & Spain & Yes & $+\mathrm{N}$ & & \\
\hline Dadvand $^{66}$ & 253 & No & Spain & No & & $+\mathrm{N}$ & \\
\hline Dzhambov $^{72}$ & 112 & No & Bulgaria & No & + & $+\mathrm{N}$ & \\
\hline De Keijzer ${ }^{55}$ & 6506 & Yes & UK & Yes & $+\mathrm{N}$ & & \\
\hline Flouri $^{56}$ & 4758 & Yes & UK & No & + & & \\
\hline Hystad $^{57}$ & 6658 & Yes & Canada & No & $-N$ & & \\
\hline Kuhn $^{73}$ & 341 & No & Germany & No & & $+\mathrm{N}$ & \\
\hline $\operatorname{Liao}^{67}$ & 1312 & No & China & No & + & & \\
\hline Reuben $^{68}$ & 1658 & Yes & UK & Yes & $\mathrm{N}$ & & \\
\hline Wang $^{69}$ & 3544 & No & China & No & $\mathrm{N}$ & & \\
\hline Ward $^{70}$ & 72 & No & New Zealand & No & $\mathrm{N}$ & & \\
\hline$W^{59}$ & 2424 & Yes & UK & No & & & - \\
\hline$W u^{58}$ & 7505 & Yes & UK & No & & & $-N$ \\
\hline $\mathrm{Yu}^{71}$ & 3240 & No & China & No & $\mathrm{N}$ & & \\
\hline Yuchi $^{60}$ & 678000 & Yes & Canada & Yes & & & +- \\
\hline Zhu $^{61}$ & 6994 & Yes & China & Yes & & & + \\
\hline Zijlema ${ }^{62}$ & 1628 & Yes & $\begin{array}{l}\text { Spain, UK, } \\
\text { Netherlands }\end{array}$ & No & $+\mathrm{N}$ & & \\
\hline \multicolumn{5}{|c|}{ Studies with positive associations } & 9 & 3 & 3 \\
\hline \multicolumn{5}{|c|}{ Studies with inverse associations } & 1 & 0 & 3 \\
\hline \multicolumn{5}{|c|}{ Studies with null associations } & 12 & 3 & 1 \\
\hline \multicolumn{5}{|l|}{ Total studies } & 15 & 3 & 5 \\
\hline
\end{tabular}

${ }^{*}$ Full list of papers found in online supplemental text 1.

+, positive association; -, inverse association; N, null association.

Greater neighbourhood greenness was associated with lower risk of $\mathrm{AD},{ }^{9}$ non- $\mathrm{AD}^{60}$ and Parkinson's disease diagnoses $^{60}$ in some studies, but increased risk of cognitive impairment $^{58} 59$ and $\mathrm{AD}$ diagnoses ${ }^{60}$ in others. Greater neighbourhood greenness/green space was positively associated with intelligence, ${ }^{63}$ global cognition, ${ }^{55}$ reasoning, ${ }^{55}$ verbal fluency ${ }^{55}$ and visual attention/executive processing. ${ }^{556-64}$ In addition, greater green space (ie, forests) was associated with better amydala integrity measured via MRI. ${ }^{73}$ Null associations were found between neighbourhood greenness/green space and intelligence, ${ }^{6364}$ global cognition, ${ }^{5369}{ }^{71}$ short-term memory ${ }^{55}$ and visual attention/executive processing. ${ }^{62}$ Time spent in natural environments was not associated with visual attention/executive processing. ${ }^{62}$ Lastly, urban green space was not associated with brain integrity measured via MRI. ${ }^{73}$

\section{Findings by green space measure}

Green space definitions included: (1) greenness measured using the NDVI or Enhanced Vegetation Index (EVI) ${ }^{9} 54555760-6265-697172$; (2) tree canopy/cover measured using vegetation continuous fields (VCF) ${ }^{54}$; (3) neighbourhood percentage green/park space or park area 53565859636473 ; (4) time spent in green space (objective or self-reported) $^{6270}$; (5) self-reported amount of natural environment near residence ${ }^{62}$ and (6) distance from residence to natural outdoor environment ${ }^{62}$ (table 2). Three studies examined more than one green space measure: (1) NDVI and $\mathrm{VCF}^{54}$; (2) NDVI and $\mathrm{EVI}^{55}$; and (3) NDVI, distance to natural outdoor environment, 
Table 4 Findings by green space-brain health association investigated and author name

\begin{tabular}{|c|c|c|c|c|c|c|c|c|c|}
\hline \multirow{2}{*}{$\begin{array}{l}\text { Green space } \\
\text { measure* }^{*}\end{array}$} & \multicolumn{3}{|l|}{ Cognition } & \multicolumn{3}{|l|}{ MRI } & \multicolumn{3}{|c|}{$\begin{array}{l}\text { Diagnosis of cognitive } \\
\text { impairment/dementia }\end{array}$} \\
\hline & + & - & $\mathbf{N}$ & + & - & $\mathbf{N}$ & + & - & $\mathbf{N}$ \\
\hline $\begin{array}{l}\text { Percent green/ } \\
\text { park space }\end{array}$ & $\begin{array}{l}\text { Cherrie }^{d} \\
\text { Cherrie }^{e} \\
\text { Flouri }\end{array}$ & & $\begin{array}{l}\text { Cherrie }^{d} \\
\text { Cherrie }^{e} \\
\text { Clarke }\end{array}$ & Kuhn & & Kuhn & & $\begin{array}{l}W u^{f} \\
W u^{g}\end{array}$ & $W u^{9}$ \\
\hline
\end{tabular}

Year of publication: ${ }^{\mathrm{a}} 2015$; ${ }^{\mathrm{b}} 2017$; ${ }^{\mathrm{c}} 2018$; ${ }^{\mathrm{d}} 2018$; ${ }^{\mathrm{e}} 2019$; ${ }^{\mathrm{f}} 2015 ;{ }^{\mathrm{g}} 2017$.

${ }^{*}$ Full list of papers found in online supplemental text 1.

-, inverse association; +, positive association; N, null association; NDVI, Normalised Difference Vegetation Index.

and self-reported green space measures. ${ }^{62}$ Most studies measured green space in the residential neighbourhood, although a few additionally measured green space surrounding schools and school routes. ${ }^{64} 65$ No studies examined work area green spaces. NDVI was the most commonly used measure. The boundaries used to define green space exposures varied greatly (eg, 100-1500 m radial buffers around residences, $1000 \mathrm{~m}$ buffers around postcode centroids, US Census tracts, $50 \mathrm{~m}$ buffers around school route).

NDVI: Ten of 14 studies $^{954555760-6265-697172}$ using NDVI found associations (nine positive, ${ }^{9} 5455606165-6772$ two inverse $^{5760}$ (table 2). Of the studies with positive findings, one examined MRI brain measures ${ }^{66}$ and three examined risk/odds of cognitive impairment/dementia. ${ }^{96061}$ The remaining studies with positive findings focused on various cognitive domains. In studies with inverse associations,



Figure 2 Number of studies by country. 5-year NDVI increase was associated with worse reasoning in 40-69 years old ${ }^{57}$ and greater greenness was associated with lower risk of non-AD dementia and Parkinson's disease among $45-84$ years old. ${ }^{60}$

Park space: Two ${ }^{6364}$ of three studies on percent/amount of residential park space found positive associations with cognitive change in late life (table 2). These positive associations were restricted to childhood and mid-life park space exposures and cognitive changes from ages 70 to 76. No associations were observed between early-life and mid-life exposures and cognitive changes from ages 11 to 76 or between late-life park space exposure and cognitive changes at any age (11-76 years). The third study found no associations between neighbourhood park area and cognition. ${ }^{53}$

Other measures: Measures of time spent in green space based on objective GPS tracking ${ }^{70}$ or self-report ${ }^{62}$

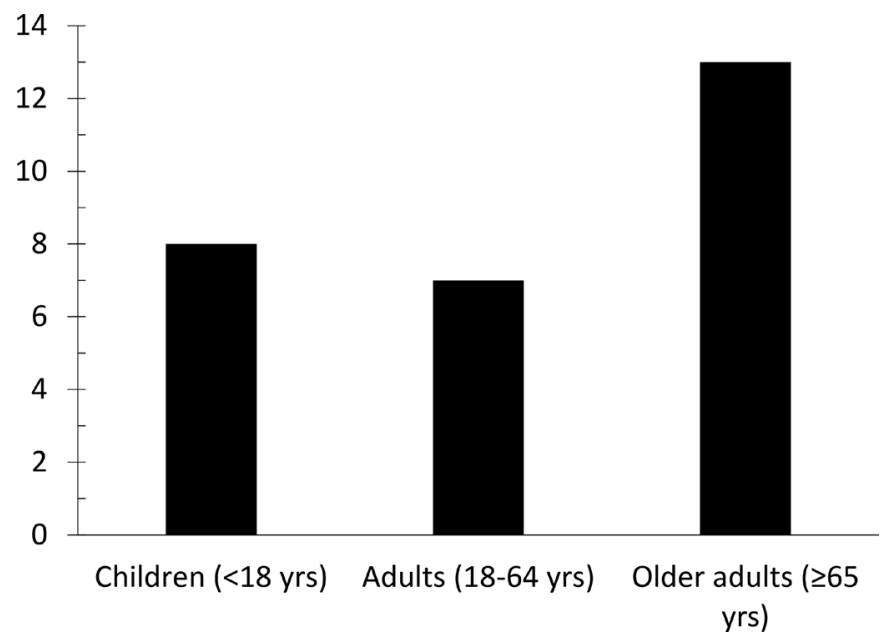

Figure 3 Number of studies by age group. 


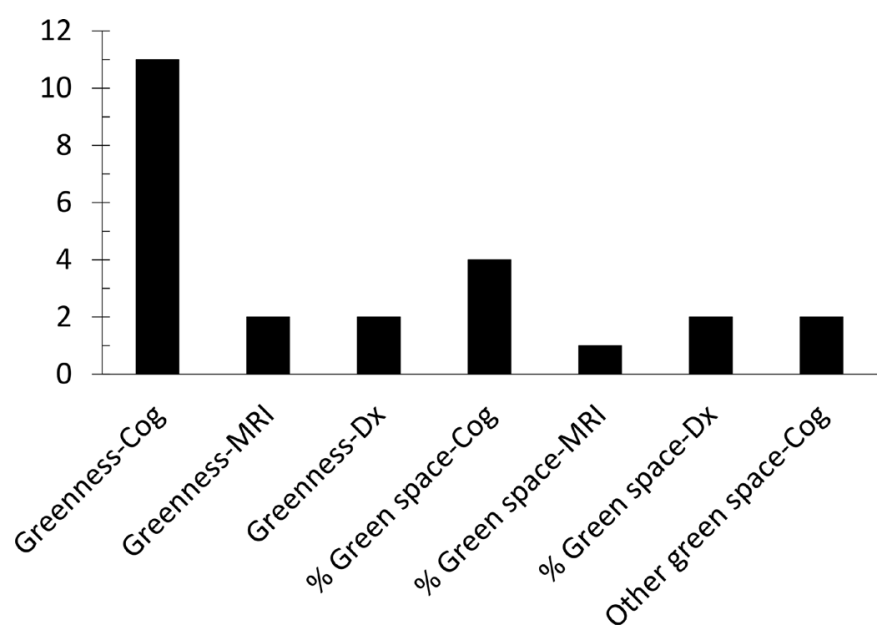

Figure 4 Number of studies by green space-brain health association. Cog, cognition; Dx, diagnosis of cognitive impairment/dementia; Greenness, measure of greenness such as Normalised Difference Vegetation Index, \% green space, per cent or amount of neighbourhood composed of green space/park space; other green space, time spent in green space, distance to nearest green/park space and/or self-reported measures.

were not associated with cognition. Positive associations were observed between percentage residential green space derived from land use data and spatial working memory, ${ }^{56}$ and between distance to the nearest natural outdoor environment and visual attention/executive processing. ${ }^{62}$ Greater amounts of forest surrounding the residence were associated with greater amygdala integrity, whereas amount of neighbourhood urban green space was not associated with MRI measures of brain integrity. ${ }^{73}$ Percentage green space and private gardens based on land use data was inversely associated with odds of cognitive impairment/dementia. ${ }^{585}$ Tree canopy/cover (VCF) was not associated with attention in children. ${ }^{54}$ Lastly, self-reported amount of residential natural environment was not associated with visual attention/executive processing. $^{62}$

\section{Findings by brain health measure}

Fifteen studies $(68 \%)$ examined cognitive function. ${ }^{53-57}$ 62-65 67-72 A range of cognitive domains were assessed, including but not limited to global cognition, working memory, attention, reasoning, verbal fluency and executive function. Five studies (23\%) (585961 $6971^{\text {used }}$ the Mini Mental State Exam, a global cognition screening test, while the remaining used a variety of other instruments. Five studies $(23 \%)^{958-61}$ examined diagnosis of cognitive impairment or dementia (including $\mathrm{AD}$ and Parkinson disease) and three focused on brain MRI. ${ }^{66} 7273$ Eight studies $(36 \%)^{5455606163-6568}$ used longitudinal data on brain health, but only five $(23 \%)^{556063-65}$ actually examined longitudinal changes in brain health (ie, cognitive decline or dementia risk).

Ten studies (45\%) found associations between green space and cognition (nine positive, ${ }^{54-56} 62-656772$ one inverse $^{57}$ ) (table 3). Greater greenness/green space was associated with global cognition, working memory, spatial working memory attention, visual attention, reasoning, fluency and measures of intelligence and childhood intellectual development, as delineated in the sections further above. The three studies using brain MRI found positive associations between greenness/green space and certain brain regions, ${ }^{66}$ cortical thickness ${ }^{72}$ and amygdala integrity. ${ }^{73}$ Two studies found positive associations between greenness/green space and $\mathrm{AD},{ }^{9}$ non-Alzheimer's dementia $^{60}$ and Parkinson's disease ${ }^{60}$ diagnoses, whereas three found inverse associations with $\mathrm{AD}^{60}$ or cognitive impairment/dementia diagnoses. 5859

\section{Effect modification}

Effect modification is variation in the association between an exposure and outcome depending on the value of another factor. Seven 55575861636467 of 11 studies $^{9} 54-5861$ 63-65 67 found effect modification (online supplemental table 4). Green space-brain health associations were stronger in/limited to women ${ }^{555763}$; APOE $\varepsilon 4$ non-carriers ${ }^{6163}$ and those with lower occupational class, ${ }^{63}$ higher education levels, ${ }^{55}$ lower body mass index ${ }^{67}$ and younger age $^{61}$ (in study of older adults). Associations also were stronger among residents of conurbations ${ }^{58}$ (urbanised area comprised of multiple cities/towns), areas with lower traffic accident densities ${ }^{64}$ and areas of higher deprivation. ${ }^{55}$ Other studies found no effect modification by neighbourhood SES, ${ }^{95665}$ sex, ${ }^{64}$ maternal education, ${ }^{65}$ residential stability/years in residence, ${ }^{56}$ race,${ }^{57}$ marital status, ${ }^{57}$ city $^{57}$ or household income. ${ }^{57}$

\section{Mediation}

Three $^{657172}$ of seven studies ${ }^{53556265677172}$ suggested mediation, which is the presence of an intermediary variable associated with both the exposure and outcome that potentially explains the causal mechanism linking the two variables (online supplemental table 4). Traffic-related air pollution (elemental carbon in residence) mediated associations between school greenness and working memory and attentiveness in children ${ }^{65}$ and self-reported physical activity mediated associations between greater residential greenness and global cognition in older adults. ${ }^{71}$ Associations between greater neighbourhood greenness and better global cognition among middle-aged adults were mediated by lower waist circumference but not by systolic blood pressure, total cholesterol, glucose, air pollution $\left(\mathrm{NO}_{2}\right)$ or traffic-related noise. ${ }^{72}$ The other four studies found no mediation of green space-brain health associations by physical activity, ${ }^{53} 55$ social measures (eg, interaction, loneliness), ${ }^{53}$ perceived mental health, ${ }^{62}$ traffic noise annoyance, ${ }^{62}$ worry about air pollution ${ }^{62}$ or air pollution levels (ie, $\mathrm{PM}_{2.5}$ ). ${ }^{55}$

\section{DISCUSSION}

Evidence was found for associations between green space exposure measured at various life stages and brain health. Seventy-one per cent of NDVI studies (greenness) found 
positive associations. Greater neighbourhood greenness/ green space was positively associated with multiple cognitive domains, brain regions and lower odds/risk of $\mathrm{AD}$ and non-AD dementia. However, some studies found inverse or null associations, few studies were conducted within each major age group, and the studies employed limited and heterogeneous methods and definitions. The remainder of this section focuses on the second aim of the scoping review, which is to identify scientific gaps for future research.

\section{Brain health measures}

The diversity of employed brain health measures limits study comparisons. Measures of attention were associated with green space in more than one study, ${ }^{546265}$ but additional research is needed to confirm these associations. Studies more frequently assessed executive function, attention, and working memory, and fewer examined short-term or long-term memory, language/ fluency, processing speed or visuospatial function. The focus on the former cognitive domains may be due to data availability, but also potential hypothesised underlying mechanisms relating green space and brain health, in which green space exposure restores attention and reduces mental fatigue/stress. ${ }^{26-28}$ Nonetheless, green space exposures may be associated with other cognitive measures reflecting brain regions susceptible to green space-related behaviours/exposures. New studies are needed to assess green space associations with cognitive domains commonly affected in typical and atypical $\mathrm{AD}$ presentations, including episodic memory, ${ }^{15}$ visuospatial processing ${ }^{74}$ and language. ${ }^{75}$ These cognitive domains have been associated with physical activity, social engagement and air pollution exposure ${ }^{76-78}$ and are important to investigate in future studies given the plausible mechanisms relating green spaces and these health behaviours/ exposures (as detailed in introduction).

Greater greenness/green space displayed mixed associations (positive/inverse) with diagnoses of cognitive impairment or dementia. The mixed findings may be explained by the employed study methods, as three of the four studies were cross-sectional and none examined or controlled for early-life and mid-life factors beyond educational attainment. With the onset of health problems or cognitive symptoms, individuals may be more likely to move to greener rural and suburban areas where there are assisted living and nursing care residences. Thus, the associations between greater late-life neighbourhood greenness/green space and increased odds/ risk of cognitive impairment may be explained by reverse causality/self-selection into greener neighbourhoods in later life. Reverse causality will need to be ruled out in future studies by using more sophisticated study designs and methods (eg, life course, instrumental variables).

Clinical diagnoses may be biased by cultural or education factors that may increase or decrease the chance of receiving a diagnosis irrespective of disease presence. For instance, minorities may be more likely to receive dementia diagnoses if educational and cultural differences are unaccounted for in cognitive testing or if a higher prevalence of comorbidities increases ADRD risk. ${ }^{79}$ Nevertheless, diagnoses are clinically significant measures of brain health, particularly when made by specialists with expertise in discerning the presence and aetiology of dementia, and thus are useful measures for future green space-brain health research in older adults.

To date, three studies investigated associations between green space and MRI biomarkers, specifically regional brain volumes, ${ }^{66}$ measures of structural integrity ${ }^{73}$ and measures of cortical thickness ${ }^{72}$ obtained from structural MRI. The study of associated brain regions ${ }^{66}$ used an intensive method of analysis (examining associations for each three-dimensional pixel (voxel) of brain image) that significantly limited the number of confounders included in the multivariable analyses. An alternative to the voxelwise analysis, which would allow controlling for multiple important confounders, would be to measure brain health/atrophy using regional brain volumes $\left(\mathrm{mm}^{3}\right)$ and cortical thickness determined through standardised segmentation techniques. ${ }^{80}$ The findings for associations between greater greenness/green space and greater amygdala integrity and cortical thickness will need to be replicated. Lastly, measures of global brain atrophy from MRI, such as total grey matter volume or ventricular volume, may be a useful addition for future studies under the presumption that green space exposures affect overall brain development and ageing.

\section{Green space measures}

This review suggests that NDVI is a valuable measure for future studies of green space and brain health. However, NDVI does not assess tree canopy/cover or other qualities of green spaces (eg, park amenities).$^{20}$ Future work will need to consistently incorporate quality measures including tree canopy/cover, availability of park amenities (eg, walking trails), and safe walking routes/sidewalks, which will help identify types of green space environments ${ }^{20}$ most effective at promoting brain health.

Studies measuring percentage of the neighbourhood composed of green space (ie, parks) found positive, ${ }^{56636473}$ inverse $^{5859}$ and null associations, ${ }^{53} 58636473$ warranting additional studies. Compared with NDVI (greenness), percentage green space may better capture access to green spaces. For instance, associations with NDVI measures can be affected by the chosen cut points to define healthy vegetation (eg, NDVI $>0.40$ or NDVI $>0.60$ ), the satellite image used to derive NDVI (affected by season and cloud cover), or green space fragmentation (pockets) that can skew mean NDVI values. ${ }^{20}$ Green space access may be a stronger predictor of healthy behaviours such as physical activity, particularly among socioeconomically disadvantaged individuals with limited resources and opportunities for exercise. ${ }^{81}$ Other measures of green space access to should be investigated (eg, number of neighbourhood parks) to determine the strongest predictors of both healthy behaviours and better brain health. 
The single study incorporating self-reported measures of green space exposure found no associations. ${ }^{62}$ Objective green space measures are necessary to suggest target amounts and qualities of green space for interventions, plans and policies. However, self-reported and perceived measures may be useful in tandem with objective measures. Valid and reliable green space questionnaires would minimise burden and data security concerns in attempting to derive objective measures from residential addresses across the life span.

The majority of studies did not measure actual exposure to green spaces (ie, time spent in green spaces). ${ }^{62} 70$ Travel diaries could be used to assess time spent in green spaces, although compliance in diary completion and misreporting may be an issue. ${ }^{82}$ Although studies have successfully incorporated GPS to investigate neighbourhood environmental exposures and outcomes including physical activity, ${ }^{83-85}$ costs, difficulty in recruiting, participant time required and non-compliance can be a hurdle. ${ }^{86}$ Despite these limitations, GPS and travel diary measures of time spent in green space provide increased specificity of exposure needed to make informed decisions about green space-brain health associations. If individuals live in neighbourhoods with greater access to green space but they do not regularly spend time in those spaces, then associations with brain health observed in prior research have been spurious or biased by residual confounding.

Places for estimating green space exposures may depend on the age group under study, but only two studies measured non-residential exposures. ${ }^{64} 65$ Green space exposure may occur most frequently in residential and school environments among children; residential, working and recreational environments among working adults; and residential and recreational environments among older adults. ${ }^{6484}$ Future studies will benefit from a more comprehensive assessement of places for green space exposures, and longitudinal studies following individuals progressing through these life stages should keep age-based differences in activity spaces in mind.

\section{Life course exposures}

Many of the studies of middle-aged and older-aged adults were cross-sectional ${ }^{93357-5961626971-73}$ and lacked consideration of earlier life green space exposures. ${ }^{9} 53557-6269$ 71-73 Childhood exposures may be most critical for determining late-life brain health by influencing healthy brain development. These neurodevelopmental benefits may impart cognitive reserve and resilience through older ages, which protects against ADRD neuropathology and resists symptoms despite neuropathology ${ }^{87}$ Green space exposure patterns during childhood may also establish healthy habits including physical activity that continue through adulthood to boost and maintain brain health. The importance of including childhood measures in future studies also applies to confounders such as earlylife personal and neighbourhood SES, which have been found to be associated with late-life cognitive health. ${ }^{88}$
Some evidence suggests that mid-life behaviours may be stronger predictors of late-life cognitive decline and dementia risk than late-life behaviours. ${ }^{89} 90$ In a similar fashion, green space exposures in mid-life versus latelife may be more strongly associated with late-life brain health. Mid-life exposures are of particular interest because the neuropathology associated with ADRD often starts decades prior to symptom development (ie, in midlife). ${ }^{91}$ During mid-life, green space-related behaviours/ exposures such as physical activity may help resist the development of ADRD neuropathology or decrease the neuropathological burden. ${ }^{92}$ Yet, even late-life green space exposures may help maintain brain health in older age by providing accessible places that encourage exercise, relaxation, and socialising. Life course studies are needed to determine the critical periods of green space exposure related to late-life brain health and ADRD risk.

\section{Causal mechanisms}

Traffic-related air pollution and self-reported physical activity were found to be mediators, providing preliminary evidence for these two causal mechanisms. Future evaluation of mediation by physical activity should use rigorous, objective measures such as those obtained from accelerometers. Social engagement and related measures were not found to be mediators, and mental health (eg, anxiety, depression) and immune function were not examined in any study. Altogether, few studies examined mediation, additional work is need to determine causal pathways for green space-brain health associations and future studies will need to employ rigorous methods to evaluate mediation. ${ }^{93}$

\section{Future research directions}

New studies will need to incorporate longitudinal measures of green space (accumulation of exposures and changes over time) and brain health. GPS-based measures of green activity spaces and time spent in green spaces will improve the quantification and quality assessment of green space exposures. Use of brain biomarkers such as structural and functional MRI, PET scans and CSF biomarkers to detect brain neurodegeneration/ADRD may provide biological evidence for associations. Green space exposures should temporally precede the brain health measures, and the validity and reliability of green space measures need to be established. Causal mechanisms need to be delineated through rigorous investigation of potential mediators. In addition, the evidence base will be strengthened by capitalising on natural experiments (eg, planned green space additions) to study green space associations with brain health.

Future studies will need to incorporate relevant factors insufficiently examined to date, including the potential impact of residential moves, seasonality of exposure/regional climate, bias due to self-selection into greener neighbourhoods (ie, reverse causality) and neighborhood-level confounders (eg, crime, population density). Research is needed on the pertinent places (eg, 
neighbourhood, work, recreational) and boundaries (eg, $1000 \mathrm{~m}$ buffer) for green space exposures. Studies need to determine if associations are present irrespective of or instead depend on race/ethnicity and culture, by demonstrating associations in multiple international contexts and in various regions of diverse countries such as the USA.

\section{LIMITATIONS}

Limitations of the reviewed studies include lack of consideration of early-life green space exposures and examination of actual time spent in green spaces. Thus, the studies were likely affected by misclassification/information bias. Selection bias was also likely for many of the studies that restricted to samples with non-missing data on exposures and outcomes.

This review may be limited by positive publication bias. In addition, papers may have been missed due to the nature of this rapid review, which was based on three databases, a restricted review of the Web of Science search results (detailed in the methods section and online supplemental figure 1), and a single reviewer. However, the review of reference lists and related reviews helped reduce the possibility. As this was a rapid review with scoping aims, ${ }^{47} 495152$ it was never intended to systematically evaluate the evidence for risk of bias, which will be reserved for future systematic reviews.

\section{CONCLUSION}

This rapid review identified twenty-two studies of green space and brain health. The majority of studies were cross-sectional and the green space and brain health measures were heterogeneous. Despite the limitations, multiple studies investigating neighbourhood greenness found positive associations with brain health outcomes at various life stages. Thus, the evidence is suggestive that green space is associated with brain health and future systematic reviews are warranted. The observed positive associations need to be replicated in longitudinal and life course studies of diverse cohorts and in studies using more rigorous measurements and statistical methods. These improvements are needed to build a case for communitylevel green space interventions to impart brain resilience, maintain/improve cognition and reduce ADRD risk in late life.

Contributors LB is the sole author of this paper and as such completed all of the work, including data acquisition and interpretation, drafting and revising the paper for intellectual content, final approval of the version to be published, and agreement to be accountable for all aspects of the work.

Funding LB is supported by National Institutes of Health/National Institute on Aging award K01AG063895.

Competing interests None declared.

Patient consent for publication Not required.

Provenance and peer review Not commissioned; externally peer reviewed.
Data availability statement Data sharing not applicable as no datasets generated and/or analysed for this study. Deidentified data (ie, search results from the databases) are available on request of LB (lbesser@fau.edu). Any reuse or sharing of these data require LB's approval.

Supplemental material This content has been supplied by the author(s). It has not been vetted by BMJ Publishing Group Limited (BMJ) and may not have been peer-reviewed. Any opinions or recommendations discussed are solely those of the author(s) and are not endorsed by BMJ. BMJ disclaims all liability and responsibility arising from any reliance placed on the content. Where the content includes any translated material, BMJ does not warrant the accuracy and reliability of the translations (including but not limited to local regulations, clinical guidelines, terminology, drug names and drug dosages), and is not responsible for any error and/or omissions arising from translation and adaptation or otherwise.

Open access This is an open access article distributed in accordance with the Creative Commons Attribution Non Commercial (CC BY-NC 4.0) license, which permits others to distribute, remix, adapt, build upon this work non-commercially, and license their derivative works on different terms, provided the original work is properly cited, appropriate credit is given, any changes made indicated, and the use is non-commercial. See: http://creativecommons.org/licenses/by-nc/4.0/.

\section{ORCID iD}

Lilah Besser http://orcid.org/0000-0001-9945-0877

\section{REFERENCES}

1 Frumkin H, Bratman GN, Breslow SJ, et al. Nature contact and human health: a research agenda. Environ Health Perspect 2017;125:075001.

2 Hartig T, Mitchell R, de Vries S, et al. Nature and health. Annu Rev Public Health 2014;35:207-28.

3 Twohig-Bennett C, Jones A. The health benefits of the great outdoors: a systematic review and meta-analysis of greenspace exposure and health outcomes. Environ Res 2018;166:628-37.

4 Gascon M, Triguero-Mas M, Martínez D, et al. Residential green spaces and mortality: a systematic review. Environ Int 2016;86:60-7.

5 de Keijzer C, Gascon M, Nieuwenhuijsen MJ, et al. Long-Term green space exposure and cognition across the life course: a systematic review. Curr Environ Health Rep 2016;3:468-77.

6 Fong KC, Hart JE, James P. A review of epidemiologic studies on Greenness and health: updated literature through 2017. Curr Environ Health Rep 2018;5:77-87.

7 Vanaken G-J, Danckaerts M. Impact of Green Space Exposure on Children's and Adolescents' Mental Health: A Systematic Review. Int $J$ Environ Res Public Health 2018;15:2668.

8 Hankey S, Marshall JD. Urban form, air pollution, and health. Curr Environ Health Rep 2017;4:491-503.

9 Brown S, Perrino T, Lombard J, et al. Health disparities in the relationship of neighborhood Greenness to mental health outcomes in 249,405 U.S. Medicare beneficiaries. Int J Environ Res Public Health 2018;15:430.

10 Roberts R, Knopman DS. Classification and epidemiology of $\mathrm{MCl}$. Clin Geriatr Med 2013;29:753-72.

11 World Health Organization. Dementia. Available: https://www.who.int/ news-room/fact-sheets/detail/dementia [Accessed 02 Oct 2020].

12 Baumgart M, Snyder HM, Carrillo MC, et al. Summary of the evidence on modifiable risk factors for cognitive decline and dementia: a population-based perspective. Alzheimers Dement 2015;11:718-26.

13 Jack CR, Bennett DA, Blennow K, et al. NIA-AA research framework: toward a biological definition of Alzheimer's disease. Alzheimers Dement 2018;14:535-62.

14 Montine TJ, Monsell SE, Beach TG, et al. Multisite assessment of NIA-AA guidelines for the neuropathologic evaluation of Alzheimer's disease. Alzheimers Dement 2016;12:164-9.

15 Hugo J, Ganguli M. Dementia and cognitive impairment: epidemiology, diagnosis, and treatment. Clin Geriatr Med 2014;30:421-42.

16 Apostolova LG, Green AE, Babakchanian S, et al. Hippocampal atrophy and ventricular enlargement in normal aging, mild cognitive impairment (MCl), and Alzheimer disease. Alzheimer Dis Assoc Disord 2012;26:17-27.

17 Liu S, Liu J, Wang X-D, et al. Caregiver burden, sleep quality, depression, and anxiety in dementia caregivers: a comparison of frontotemporal lobar degeneration, dementia with Lewy bodies, and Alzheimer's disease. Int Psychogeriatr 2018;30:1131-8. 
18 Galvin JE, Howard DH, Denny SS, et al. The social and economic burden of frontotemporal degeneration. Neurology 2017;89:2049-56.

19 Alzheimer's Association. 2020 Alzheimer's Disease Facts and Figures, 2020. Available: https://www.alz.org/media/Documents/ alzheimers-facts-and-figures.pdf [Accessed 05 Aug 2020].

20 Markevych I, Schoierer J, Hartig T, et al. Exploring pathways linking greenspace to health: theoretical and methodological guidance. Environ Res 2017;158:301-17.

21 Erickson KI, Voss MW, Prakash RS, et al. Exercise training increases size of hippocampus and improves memory. Proc Natl Acad Sci U S A 2011;108:3017-22.

22 Fleischman DA, Yang J, Arfanakis K, et al. Physical activity, motor function, and white matter hyperintensity burden in healthy older adults. Neurology 2015;84:1294-300.

23 Vivar C, Potter MC, van Praag H. All about running: synaptic plasticity, growth factors and adult hippocampal neurogenesis. Curr Top Behav Neurosci 2013;15:189-210.

24 van Tol M-J, van der Wee NJA, van den Heuvel OA, et al. Regional brain volume in depression and anxiety disorders. Arch Gen Psychiatry 2010;67:1002-11.

25 Wilker EH, Preis SR, Beiser AS, et al. Long-Term exposure to fine particulate matter, residential proximity to major roads and measures of brain structure. Stroke 2015;46:1161-6.

26 Berto R. The role of nature in coping with Psycho-physiological stress: a literature review on restorativeness. Behav Sci 2014;4:394-409.

27 Stevenson MP, Schilhab T, Bentsen P. Attention restoration theory II: a systematic review to clarify attention processes affected by exposure to natural environments. $J$ Toxicol Environ Health B Crit Rev 2018;21:227-68.

28 Pasanen T, Johnson K, Lee K, et al. Can nature walks with psychological tasks improve mood, self-reported restoration, and sustained attention? results from two experimental field studies. Front Psychol 2018;9:2057.

29 Stigsdotter UK, Ekholm O, Schipperijn J, et al. Health promoting outdoor environments--associations between green space, and health, health-related quality of life and stress based on a Danish national representative survey. Scand J Public Health 2010;38:411-7.

30 Van Den Berg AE, Custers MHG. Gardening promotes neuroendocrine and affective restoration from stress. $J$ Health Psychol 2011;16:3-11

31 Kessing LV. Depression and the risk for dementia. Curr Opin Psychiatry 2012;25:457-61.

32 Ruokolainen L, Hertzen L, Fyhrquist N, et al. Green areas around homes reduce atopic sensitization in children. Allergy 2015;70:195-202.

33 Sun M, Ma K, Wen J, et al. A Review of the Brain-Gut-Microbiome Axis and the Potential Role of Microbiota in Alzheimer's Disease. $J$ Alzheimers Dis 2020;73:849-65.

34 Nielsen CC, Gascon M, Osornio-Vargas AR, et al. Natural environments in the urban context and gut microbiota in infants. Environ Int 2020;142:105881.

35 Logan AC. Dysbiotic drift: mental health, environmental grey space, and microbiota. J Physiol Anthropol 2015;34:23.

36 Dalton AM, Wareham N, Griffin S, et al. Neighbourhood greenspace is associated with a slower decline in physical activity in older adults: a prospective cohort study. SSM Popul Health 2016;2:683-91.

37 Bancroft C, Joshi S, Rundle A, et al. Association of proximity and density of Parks and objectively measured physical activity in the United States: a systematic review. Soc Sci Med 2015;138:22-30.

38 Tan ZS, Spartano NL, Beiser AS, et al. Physical activity, brain volume, and dementia risk: the Framingham study. J Gerontol A Biol Sci Med Sci 2017;72:789-95.

39 Kirk-Sanchez NJ, McGough EL. Physical exercise and cognitive performance in the elderly: current perspectives. Clin Interv Aging 2014;9:51-62.

40 Jennings $\mathrm{V}$, Bamkole $\mathrm{O}$. The relationship between social cohesion and urban green space: an avenue for health promotion. Int $J$ Environ Res Public Health 2019;16:452.

41 Krueger KR, Wilson RS, Kamenetsky JM, et al. Social engagement and cognitive function in old age. Exp Aging Res 2009;35:45-60.

42 Marioni RE, Proust-Lima C, Amieva H, et al. Social activity, cognitive decline and dementia risk: a 20-year prospective cohort study. BMC Public Health 2015;15:1089.

43 Midouhas E, Kokosi T, Flouri E. Neighbourhood-level air pollution and greenspace and inflammation in adults. Health Place 2019;58:102167.

44 Datzmann T, Markevych I, Trautmann F, et al. Outdoor air pollution, green space, and cancer incidence in Saxony: a semi-individual cohort study. BMC Public Health 2018;18:715.
45 Peters R, Ee N, Peters J, et al. Air pollution and dementia: a systematic review. J Alzheimers Dis 2019;70:S145-63.

46 Block ML, Calderón-Garcidueñas L. Air pollution: mechanisms of neuroinflammation and CNS disease. Trends Neurosci 2009;32:506-16.

47 Grant MJ, Booth A. A typology of reviews: an analysis of 14 review types and associated methodologies. Health Info Libr J 2009;26:91-108.

48 Arksey H, O'Malley L. Scoping studies: towards a methodological framework. Int J Soc Res Methodol 2005;8:19-32.

49 Peters MDJ, Godfrey CM, Khalil H, et al. Guidance for conducting systematic scoping reviews. Int J Evid Based Healthc 2015;13:141-6.

50 Zhang J, Yu Z, Zhao B, et al. Links between green space and public health: a bibliometric review of global research trends and future prospects from 1901 to 2019. Environ. Res. Lett. 2020;15:063001. ARTN 063001

51 Munn Z, Peters MDJ, Stern C, et al. Systematic review or scoping review? guidance for authors when choosing between a systematic or scoping review approach. BMC Med Res Methodol 2018;18:143.

52 Tricco AC, Antony J, Zarin W, et al. A scoping review of rapid review methods. BMC Med 2015;13:224.

53 Clarke PJ, Ailshire JA, House JS, et al. Cognitive function in the community setting: the neighbourhood as a source of "cognitive reserve'? J Epidemiol Community Health 2012;66:730-6.

54 Dadvand P, Tischer C, Estarlich M, et al. Lifelong residential exposure to green space and attention: a population-based prospective study. Environ Health Perspect 2017;125:097016.

55 de Keijzer C, Tonne C, Basagaña X, et al. Residential surrounding Greenness and cognitive decline: a 10-year follow-up of the Whitehall II cohort. Environ Health Perspect 2018;126:077003.

56 Flouri E, Papachristou E, Midouhas E. The role of neighbourhood greenspace in children's spatial working memory. Br J Educ Psychol 2019;89:359-73.

57 Hystad P, Payette Y, Noisel N, et al. Green space associations with mental health and cognitive function: results from the Quebec CARTaGENE cohort. Environ Epidemiol 2019;3:e040.

$58 \mathrm{Wu}$ Y-T, Prina AM, Jones A, et al. The built environment and cognitive disorders: results from the cognitive function and ageing study II. Am $J$ Prev Med 2017;53:25-32.

$59 \mathrm{Wu}$ Y-T, Prina AM, Jones AP, et al. Community environment, cognitive impairment and dementia in later life: results from the cognitive function and ageing study. Age Ageing 2015;44:1005-11.

60 Yuchi W, Sbihi H, Davies H, et al. Road proximity, air pollution, noise, green space and neurologic disease incidence: a population-based cohort study. Environ Health 2020;19:8.

61 Zhu A, Yan L, Shu C, et al. Apoe $\varepsilon 4$ modifies effect of residential Greenness on cognitive function among older adults: a longitudinal analysis in China. Sci Rep 2020;10:82.

62 Zijlema WL, Triguero-Mas M, Smith G, et al. The relationship between natural outdoor environments and cognitive functioning and its mediators. Environ Res 2017;155:268-75.

63 Cherrie MPC, Shortt NK, Mitchell RJ, et al. Green space and cognitive ageing: a retrospective life course analysis in the Lothian birth cohort 1936. Soc Sci Med 2018;196:56-65.

64 Cherrie M, Shortt N, Ward Thompson C, et al. Association between the activity space exposure to Parks in childhood and adolescence and cognitive aging in later life. Int J Environ Res Public Health 2019;16:632.

65 Dadvand P, Nieuwenhuijsen MJ, Esnaola M, et al. Green spaces and cognitive development in primary schoolchildren. Proc Natl Acad Sci U S A 2015;112:7937-42.

66 Dadvand P, Pujol J, Macià D, et al. The association between lifelong Greenspace exposure and 3-dimensional brain magnetic resonance imaging in Barcelona schoolchildren. Environ Health Perspect 2018;126:027012.

67 Liao J, Zhang B, Xia W, et al. Residential exposure to green space and early childhood neurodevelopment. Environ Int 2019;128:70-6.

68 Reuben A, Arseneault L, Belsky DW, et al. Residential neighborhood greenery and children's cognitive development. Soc Sci Med 2019;230:271-9

69 Wang D, Lau KK-L, Yu R, et al. Neighbouring green space and mortality in community-dwelling elderly Hong Kong Chinese: a cohort study. BMJ Open 2017;7:e015794.

70 Ward JS, Duncan JS, Jarden A, et al. The impact of children's exposure to greenspace on physical activity, cognitive development, emotional wellbeing, and ability to appraise risk. Health Place 2016;40:44-50.

71 Yu R, Wang D, Leung J, et al. Is neighborhood green space associated with less frailty? Evidence from the Mr. and MS. os (Hong Kong) study. J Am Med Dir Assoc 2018;19:528-34. 
72 Dzhambov AM, Bahchevanov KM, Chompalov KA, et al. A feasibility study on the association between residential greenness and neurocognitive function in middle-aged Bulgarians. Arh Hig Rada Toksikol 2019;70:173-85.

73 Kühn S, Düzel S, Eibich P, et al. In search of features that constitute an "enriched environment" in humans: Associations between geographical properties and brain structure. Sci Rep 2017;7:11920.

74 Wong B, Lucente DE, MacLean J, et al. Diagnostic evaluation and monitoring of patients with posterior cortical atrophy. Neurodegener Dis Manag 2019;9:217-39.

75 Rogalski E, Sridhar J, Rader B, et al. Aphasic variant of Alzheimer disease: clinical, anatomic, and genetic features. Neurology 2016;87:1337-43

76 Krell-Roesch J, Vemuri P, Pink A, et al. Association between mentally stimulating activities in late life and the outcome of incident mild cognitive impairment, with an analysis of the APOE $\varepsilon 4$ genotype. JAMA Neurol 2017;74:332-8.

77 Cleary EG, Cifuentes M, Grinstein G, et al. Association of lowlevel ozone with cognitive decline in older adults. J Alzheimers Dis 2018;61:67-78.

78 Podewils LJ, Guallar E, Kuller LH. Physical activity, APOE genotype, and dementia risk: findings from the cardiovascular health cognition study. Am J Epidemiol 2005;161:639-51.

79 Chin AL, Negash S, Hamilton R. Diversity and disparity in dementia: the impact of ethnoracial differences in Alzheimer disease. Alzheimer Dis Assoc Disord 2011;25:187-95.

80 Ledig C, Schuh A, Guerrero R, et al. Structural brain imaging in Alzheimer's disease and mild cognitive impairment: biomarker analysis and shared morphometry database. Sci Rep 2018;8:11258.

81 Besser L, Mitsova D. Associations between neighborhood green land cover and neighborhood-based walking among US older adults. Am J Prev Med 2021.

82 Frost R, McClurg D, Brady M, et al. Optimising the validity and completion of adherence diaries: a multiple case study and randomised crossover trial. Trials 2016;17:489.
83 Evenson KR, Wen F, Hillier AMY, et al. Assessing the contribution of Parks to physical activity using global positioning system and accelerometry. Med Sci Sports Exerc 2013;45:1981-7.

84 Hirsch JA, Winters M, Clarke P, et al. Generating GPs activity spaces that shed light upon the mobility habits of older adults: a descriptive analysis. Int J Health Geogr 2014;13:51.

85 James P, Hart JE, Hipp JA, et al. GPS-Based exposure to Greenness and Walkability and Accelerometry-Based physical activity. Cancer Epidemiol Biomarkers Prev 2017;26:525-32.

86 Zenk SN, Matthews SA, Kraft AN, et al. How many days of global positioning system (GPs) monitoring do you need to measure activity space environments in health research? Health Place 2018;51:52-60.

87 Montine TJ, Cholerton BA, Corrada MM, et al. Concepts for brain aging: resistance, resilience, reserve, and compensation. Alzheimers Res Ther 2019;11:22.

88 Letellier N, Gutierrez L-A, Carrière I, et al. Sex-Specific association between neighborhood characteristics and dementia: the Three-City cohort. Alzheimers Dement 2018:14:473-82.

89 Anstey KJ, Cherbuin N, Budge M, et al. Body mass index in midlife and late-life as a risk factor for dementia: a meta-analysis of prospective studies. Obes Rev 2011;12:e426-37.

90 Joas E, Bäckman K, Gustafson D, et al. Blood pressure trajectories from midlife to late life in relation to dementia in women followed for 37 years. Hypertension 2012;59:796-801.

91 Irwin K, Sexton C, Daniel T, et al. Healthy aging and dementia: two roads diverging in midlife? Front Aging Neurosci 2018;10:275.

92 Brown BM, Peiffer J, Rainey-Smith SR. Exploring the relationship between physical activity, beta-amyloid and tau: a narrative review. Ageing Res Rev 2019;50:9-18.

93 Dzhambov AM, Browning MHEM, Markevych I, et al. Analytical approaches to testing pathways linking greenspace to health: a scoping review of the empirical literature. Environ Res 2020;186:109613. 